\title{
Porcine ubiquitin-like protein MNSF $\beta$ promotes cell apoptosis and covalently binds to BCL-G to enhance staurosporine-induced apoptosis
}

\author{
Pengyuan Chen ${ }^{1 \#}$, Jin Wang ${ }^{2 \#}$, Xingye Wang ${ }^{3}$, Yaping Wang ${ }^{3}$, Chengjie Xu ${ }^{1}$, Chengjie Ji ${ }^{2}$ \\ ${ }^{1}$ Sichuan Academy of Medical Sciences \& Sichuan Provincial People's Hospital, Chengdu, China; ${ }^{2}$ Department of Laboratory Medicine, The People's \\ Hospital of Jianyang City, Jianyang, China; ${ }^{3}$ College of Veterinary Medicine, Northwest A\&F University, Yangling, China \\ Contributions: (I) Conception and design: P Chen, J Wang; (II) Administrative support: C Xu, C Ji; (III) Provision of study materials: X Wang, \\ Y Wang; (IV) Collection and assembly of data: P Chen, J Wang, X Wang, Y Wang; (V) Data analysis and interpretation: P Chen, J wang; (VI) \\ Manuscript writing: All authors; (VII) Final approval of manuscript: All authors. \\ \#These authors contributed equally to this work. \\ Correspondence to: Chengiie Ji. The People’s Hospital of Jianyang City, Jianyang, China. Email: iburningji@gmail.com; Chengjie Xu. Department \\ of Laboratory Medicine, Sichuan Provincial People's Hospital, University of Electronic Science and Technology of China, Chengdu, China. \\ Email: xuchengjie.602@163.com.
}

Background: Monoclonal non-specific suppressor factor $\beta$ (MNSF $\beta$ ) is a ubiquitously expressed member of the ubiquitin-like family. It functions as a regulator of cell apoptosis and a potential tumor suppressor, playing a vital role in the processes of immune cell function and apoptosis.

Methods: The present study constructed GFP-pMNSF $\beta$ swine umbilical vein endothelial cell (SUVEC) lines and investigated the function of porcine MNSF $\beta$ (pMNSF $\beta$ ) in apoptosis, as well as its interactions with pBCL-G. Results revealed that stably expressed pMNSF $\beta$ protein in SUVEC lines significantly enhanced staurosporine (STS)-induced apoptosis. pMNSF $\beta$ proteins interacted with pBCL-G proteins and the expression of these interacting proteins synergized to further enhance STS-induced apoptosis.

Results: GFP-pMNSF $\beta$ stably expressed SUVEC lines through transient transfection and neomycin screening methods. Over $90 \%$ of the SUVEC cultures expressed GFP signals, and $41.5 \mathrm{kDa}$ GFP-pMNSF $\beta$ proteins were detected with western blotting methods. Annexin V-PE/PI staining and flow cytometry analyses showed that overexpression of $\mathrm{pMNSF} \beta$ proteins significantly elevated STS-induced apoptosis rates. Co-immunoprecipitation methods revealed an interaction between pMNSF $\beta$ and pBCL-G proteins. BCL-G is a proapoptotic member of the BCL-2 family that has been shown to be misexpressed in human systemic lupus erythematosus, as well as mammary and prostate cancers. Here, we demonstrated that the co-expression and potential conjugation of $\mathrm{pMNSF} \beta$ and $\mathrm{pBCL}-\mathrm{G}$ proteins synergistically enhanced STSinduced apoptosis.

Conclusions: The present study was the first to characterize the function of MNSF $\beta$ in porcine cells, and to clarify the function of MNSF $\beta$ in apoptosis. These results reveal that $\mathrm{pMNSF} \beta$ is a potential molecular model for future investigations of diseases related to human MNSF $\beta$ dysfunction.

Keywords: Ubiquitin-like protein; porcine; BCL-G; swine umbilical vein endothelial cell (SUVEC); apoptosis

Submitted Aug 07, 2020. Accepted for publication Oct 20, 2020.

doi: 10.21037/atm-20-6348

View this article at: http://dx.doi.org/10.21037/atm-20-6348 


\section{Introduction}

Monoclonal non-specific suppressor factor $\beta$ (MNSF $\beta)$ is synthesized by $\mathrm{T}$ cells and is involved in various biological processes (1). MNSF $\beta$ is highly conserved, sharing a $91 \%$ homology and $<0.05$ evolutionary distance across 18 common species (2). The gene is approximately $414 \mathrm{bp}$ in length, and contains one exon encoding a 133-aminoacid product $(3,4)$. Structurally, MNSF $\beta$ protein is a fusion protein consisting of an ubiquitin-like domain fused with the ribosomal protein $\mathrm{S} 30$ at its terminus $(5,6)$.

MNSF $\beta$ has been demonstrated to function as a nonspecific suppressor of lymphokine in immune responses, and also participates in regulating eukaryotic processes, such as stress reaction, cell division, cell apoptosis, and nuclear transport (7-10). Other studies have shown that MNSF $\beta$ binds to specific lysine residues of target proteins. One well-characterized MNSF $\beta$ target is BCL-G, a proapoptotic member of the BCL-2 family, that was shown to regulate the extracellular signal-regulated kinase (ERK)/ mitogen-activated protein kinase (MAPK) signal cascade in mouse macrophage (Raw264.7) cells lines (11). Given the important roles of MNSF $\beta$ proteins, it has been studied extensively in human and mouse models $(5,12)$. However, porcine models may better serve the scientific research community because, compared to mice, they are more similar to humans in both physiology and anatomy (13-15). Thus far, the physiological significance of porcine MNSF $\beta$ ( $\mathrm{pMNSF} \beta$ ) activity in porcine models has remained elusive. Thus, studies characterizing $\mathrm{pMNSF} \beta$ gene products are critical for understanding the role of MNSF $\beta$ in human diseases.

In this study, we constructed GFP-pMNSF $\beta$ swine umbilical vein endothelial cell (SUVEC) lines and investigated the function of $\mathrm{pMNSF} \beta$ in apoptosis, as well as its interactions with porcine BCL-G (pBCL-G). Results revealed that stably expressed $\mathrm{pMNSF} \beta$ protein in SUVEC lines significantly enhanced staurosporine (STS)-induced apoptosis. $\mathrm{pMNSF} \beta$ proteins interacted with $\mathrm{pBCL}-\mathrm{G}$ proteins and the expression of these interacting proteins synergized to further enhance STS-induced apoptosis. In this study, we characterized pMNSF $\beta$, and our data suggested that MNSF $\beta$ is a potential new target for disease intervention. Additionally, results from this study can be added to the growing body of literature exploring the role of human MNSF $\beta$ in related diseases. We present the following article in accordance with the MDAR reporting checklist (available at http://dx.doi.org/10.21037/atm-20-6348).

\section{Methods}

\section{Materials}

Staurosporine was purchased from Sigma-Aldrich (St. Louis, MO, USA). Mouse monoclonal antibodies against HA and myc were purchased from Santa Cruz Biotechnology, Inc (Santa Cruz, CA, USA). Horseradish peroxidase (HRP)-conjugated goat anti-mouse secondary antibodies were obtained from Sigma-Aldrich. Both rabbit polyclonal GFP and horseradish peroxidase (HRP)conjugated goat anti-rabbit secondary antibodies were obtained from CWBiotech (Beijing, China). All experiments were conducted in compliance with current Chinese ethical legislation.

\section{Reverse transcription-polymerase chain reaction (RT-PCR)}

pMNSF $\beta$ and pBCL-G genes were cloned from porcine spleen tissue by RT-PCR. The primers used to detect pMNSF $\beta$ were as follows: PF1: 5 '-AGAGATCTATGCAGCTCTTTGTCCG-3 ' (underlining indicates the BglII site); PR 1: 5'-TAGGGCCCTTAAGAGTTGGCATT-3' (underlining indicates the ApaI site). The primer for pBCL-G were published by Jiang et al. (16). All gene products (nucleic acid and proteins) were analyzed to verify their identities with DNAStar and Blast (http://blast.ncbi.nlm.nih.gov/Blast.cgi).

\section{Plasmid constructions and transfections}

The pMNSF $\beta$ gene sequence was subcloned into the pCMV-HA and pEGFP-c1 vector to construct the recombinant plasmid pCMV-HA-pMNSF $\beta$ and pEGFPMNSF $\beta$. pCMV-myc-pBCL-G recombinant constructs were previously produced in our laboratory (16). SUVEC lines were maintained in six-well plates in Dulbecco's Modified Eagle Medium (DMEM) containing 10\% fetal bovine serum (FBS) at $37{ }^{\circ} \mathrm{C}$ with $5 \% \mathrm{CO}_{2}$. When cell densities neared $80 \%$, mediums were changed to pure DMEM, incubated for 1 hour (hr), and then DNA transfection was performed with $4 \mu \mathrm{g}$ of the recombinant plasmids per well using Lipofectamine 2000 (Invitrogen, US) according to the manufacturer's protocols.

\section{Establishment of SUVEC lines stably expressing GFP- pMNSF及 proteins}

Twenty-four hours after transfection of pEGFP-pMNSF $\beta$ 
in SUVEC lines, the culture media were changed to growth medium supplemented with $800 \mu \mathrm{g} / \mathrm{mL} \mathrm{G} 418$. After $72 \mathrm{hr}$ incubation, cells were inspected for GFP fluorescence, and the expressing cultures were trypsin $(0.25 \%)$ digested and transferred to $10 \mathrm{~cm}$ petri dishes at a density of $5 \times 10^{5}$. Every 2 days, fresh culture medium containing G418 was added. After 10-14 days of incubation, drug-resistance clonal cell populations were observed under fluorescence microscopes. Any cell populations that did not express GFP and were located near the expressing clones were removed with a $10 \mu \mathrm{L}$ pipette tip. Incubation of the clonal cultures in G418 medium continued until the fluorescent clone density reached up to $50 \%$. Fluorescence-positive clones were then transferred to 24-well plates and expanded. The cloned colonies were additionally subjected to single-cell cloning using limiting dilution methods. Finally, the clonal population exhibiting the highest fluorescence expression was selected from the first and second round of cloning for evaluation.

\section{Immunofluorescence (IF) assays}

To detect the expression of HA-tagged pMNSF $\beta$ (HApMNSF $\beta$ ), SUVEC lines were seeded into 6-well plates and transfected with pCMV-HA-pMNSF $\beta$ plasmids, using the methodologies described above. Approximately $24 \mathrm{hr}$ after transfection, the cells were fixed with $4 \%$ paraformaldehyde for 15 minutes (min) at room temperature and permeabilized with $0.2 \%$ Triton $\mathrm{X}-100 / \mathrm{PBS}$ for $5 \mathrm{~min}$. Next, cells were incubated with mouse anti-HA monoclonal antibodies $(1: 1,000)$ for $1 \mathrm{hr}$ at room temperature, followed by incubation with fluorescein isothiocyanate (FITC)conjugated goat anti-mouse immunoglobin G (IgG) secondary antibodies (Sigma-Aldrich, 1:100 dilution) for $1 \mathrm{hr}$ at room temperature. After being rinsed with phosphate-buffered saline (PBS), cells were observed under a fluorescence microscope.

\section{Western blot analyses}

Whole-cell lysates were generated using lysis buffer $(50 \mathrm{mM}$ Tris-HCl, 5 mM EDTA150 mM NaCl, 0.1\% NP-40, 0.5\% deoxycholic acid, $1 \mathrm{mM}$ sodium orthovanadate, $100 \mu \mathrm{g} / \mathrm{mL}$ PMSF) and protease inhibitors $(20 \mu \mathrm{g} / \mathrm{mL}$ leupeptin, $10 \mu \mathrm{g} / \mathrm{mL}$ pepstatin $\mathrm{A}$ and $10 \mu \mathrm{g} / \mathrm{mL}$ aprotinin). Protein concentrations of cell lysates were determined with BCA Protein Assay Kits (Pierce, USA). Equal amounts of lysates were boiled in sodium dodecyl sulfate (SDS) sample buffer for $10 \mathrm{~min}$, proteins were separated by SDSpolyacrylamide gels, and transferred onto polyvinylidene fluoride (PVDF) membranes (Millipore, USA). Membranes were incubated overnight at $4{ }^{\circ} \mathrm{C}$ in a trisbuffered saline solution containing $5 \%$ milk to block nonspecific binding sites, followed by HRP-conjugated secondary antibodies at room temperature for $1 \mathrm{hr}$. Protein bands were visualized using Luminata Classico Western HRP Substrate according to the manufacturer's instructions (Millipore, USA).

\section{Co-immunoprecipitations}

Co-immunoprecipitations were performed using Mammalian c-Myc Tag IP/Co-IP Kits (Pierce) according to the manufacturer's instructions. Briefly, SUVEC lines were transiently transfected with a mixture of pCMV-mycpBCL-G and pCMV-HA-pMNSF $\beta$ vectors, while control experiments were transfected with either a mixture of pCMV-myc-pBCL-G and pCMV-HA empty vectors, or a mixture of pCMV-HA-pMNSF $\beta$ and pCMV-myc empty vectors. Thirty-six hours post-transfection, proteins were extracted using Mammalian Protein Extraction Reagent (MPER). Samples were then incubated overnight at $4{ }^{\circ} \mathrm{C}$ in the presence of $10 \mu \mathrm{L}$ of anti-c-Myc agarose slurry. The agarose slurries were washed three times with Tris Buffered Saline plus $0.05 \%$ Tween-20, and immunoprecipitates were released from the agarose slurries by boiling in $25 \mu \mathrm{L}$ of non-reducing sample buffer for $5 \mathrm{~min}$. Western blots were carried out with anti-HA or anti-myc antibodies to detect binding partners.

\section{Statistical analyses}

Annexin V-PE/PI staining and flow cytometry method (FCM) was used to discern between apoptotic and necrotic cells death, according to the instructions. Expressions of stained cells were analyzed using double flow cytometry parameters. Resulting data were analyzed with LMD and Motilcycle software packages. Calculations of means, standard deviations (SD), and all statistical analyses were performed with SPSS (version 13.0). Differences between test and control groups were assessed using independent samples $t$-tests, with $\mathrm{P}$ values $<0.05$ considered statistically significant and $<0.01$ considered highly statistically significant. 


\section{Results}

Transfection of GFP-pMNSFß protein was stably expressed in SUVEC lines

GFP-pMNSF $\beta$ that highly expressed the clonal cell line was obtained using limiting dilution methods and G418 selection in SUVEC lines that had been transfected with the successfully constructed plasmid, pEGFP-pMNSF $\beta$, which was verified by sequencing and digestion (Figure 1A). The cloned cells expressing GFP-PMNSF protein obtained by limiting dilution continued to proliferate steadily under the treatment of G418. Figure $1 B$ shows the cell and GFP-pMNSF $\beta$ protein condition during continuous cell proliferation. As shown in Figure 1C, clonal cell lines stably expressing GFP-pMNSF $\beta$ proteins were successfully subcultured for more than 40 passages during a 4-month period. The expression of GFP-pMNSF $\beta$ proteins was analyzed by western blot at passages of 5,10 , and 40 (Figure 1D).

\section{pMNSF及 overexpression enhanced STS-induced apoptosis in SUVEC lines}

We explored the role of $\mathrm{pMNSF} \beta$ in apoptotic pathways. Apoptosis rates were quantified in stable GFP-pMNSF $\beta$ SUVEC lines induced to undergo apoptosis by exposure to STS (concentrations: 0, 100, 500, and 1,000 nM). Annexin $\mathrm{V}$-PE/PI staining in combination with FCM were used to discern between apoptotic and necrotic cells. Early apoptotic events were visualized in the lower right quadrant of FCM diagrams, whereas late apoptotic events were found in the upper right quadrant (Figure 2A). Statistical analyses of apoptosis rates, as calculated by FCM, clearly demonstrated that overexpression of $\mathrm{pMNSF} \beta$ significantly enhanced apoptosis compared to controls (Figure 2B). These results suggest that overexpressed pMNSF $\beta$ may have a similar role in apoptosis as pBCL-G, a canonical proapoptotic factor.

\section{pMNSF及 co-immunoprecipitate with pBCL-G}

In our studies, MNSF $\beta$ proteins were demonstrated to bind with BCL-G proteins. Specifically, residues in the MNSF $\beta$ $\mathrm{n}$-terminal region physically interacted with BCL-GL in humans cell lines. Previous findings indicated porcine BCL-G to be highly conserved and structurally similar to human BCL-GL, with 71\% identity between species (2). In the present study, co-immunoprecipitations were performed to determine whether pMNSF $\beta$ interacts with pBCL-G.
HA tags fused to $\mathrm{pMNSF} \beta$ and myc-BCL-G proteins were detected using western blotting techniques (Figure 3). Additionally, HA-pMNSF $\beta$ and myc-pBCL-G were overexpressed in SUVEC lines, and expression patterns were observed by indirect IF and western blotting analyses. FITC-conjugated secondary antibody signals were detected in cells expressing either myc-pBCL-G or HA-pMNSF $\beta$ proteins above background expression in parent SUVEC lines (data not shown). Furthermore, when myc-pBCL-G and HA-pMNSF $\beta$ were overexpressed in SUVEC cells, and lysates were pulled down with myc beads, HA-pMNSF $\beta$ could be detected by western blotting analyses. Conversely, HA-pMNSF $\beta$ was not detected in the immunoprecipitated product when myc-pBCL-G and HA-tag control vectors were co-expressed. Similarly, co-expressions of myc-tag empty vectors and HA-pMNSF $\beta$ yielded negative results by myc pull down methods (Figure 3). These results indicated that overexpressed $\mathrm{pMNSF} \beta$ proteins indirectly interacted with $\mathrm{pBCL}-\mathrm{G}$ proteins.

\section{Co-expression of pMNSF $\beta$ and pBCL-G significantly enhanced STS-induced apoptosis}

Our previous studies demonstrated that pBCL-G overexpression enhanced STS-induced apoptosis (16). Given that $\mathrm{pBCL}-\mathrm{G}$ and $\mathrm{pMNSF} \beta$ proteins indirectly interacted, we explored whether STS-induced apoptosis would be influenced by co-transfection of pMNSF $\beta$ and pBCL-G plasmids. Our previous work demonstrated that early apoptotic events induced by 500-1,000 nM STS were significantly increased when cells stably expressed GFP-pBCL-G (16). SUVEC cells stably expressing GFPpBCL-G were transfected with pCMV-HA-pMNSF $\beta$ or pCMV-HA empty vectors. Twenty-four hours after transfection, cells were treated with $500 \mathrm{nM}$ STS and incubated for $18 \mathrm{hr}$. Using the same FCM methods as described above, cell apoptosis was detected and quantified. Apoptosis rates were significantly elevated in cells overexpressing pBCL-G and pMNSF $\beta$, when compared with cells overexpressing pBCL-G and the empty vector alone (Figure 4).

\section{Discussion}

In this study, stably expressing GFP-pMNSF $\beta$ SUVEC lines provided useful and convenient molecular tools. Previous studies have demonstrated MNSF $\beta$ to be a natural immunosuppressive factor that is involved in various 
A

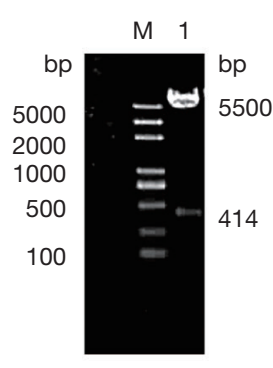

B
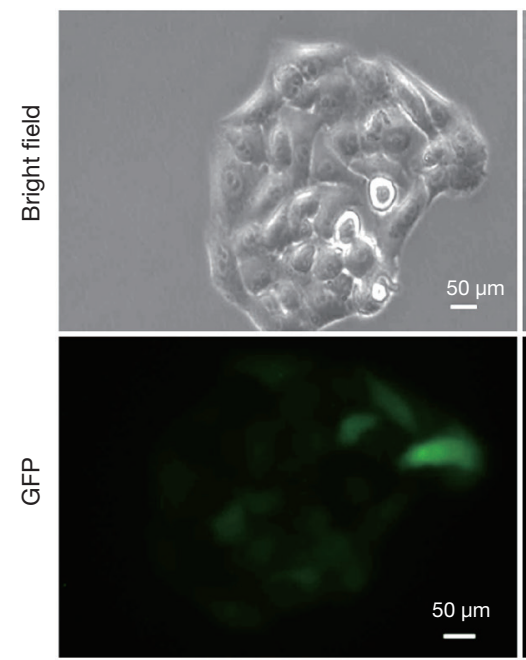

Day 6
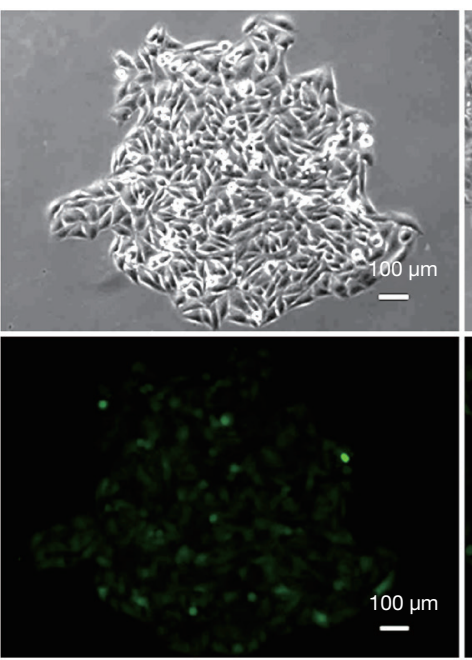

Day 10

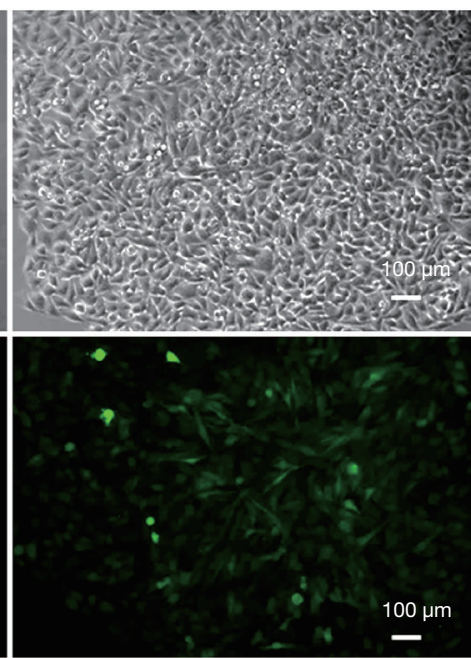

C GFP-pMNSF $\beta$ Mock
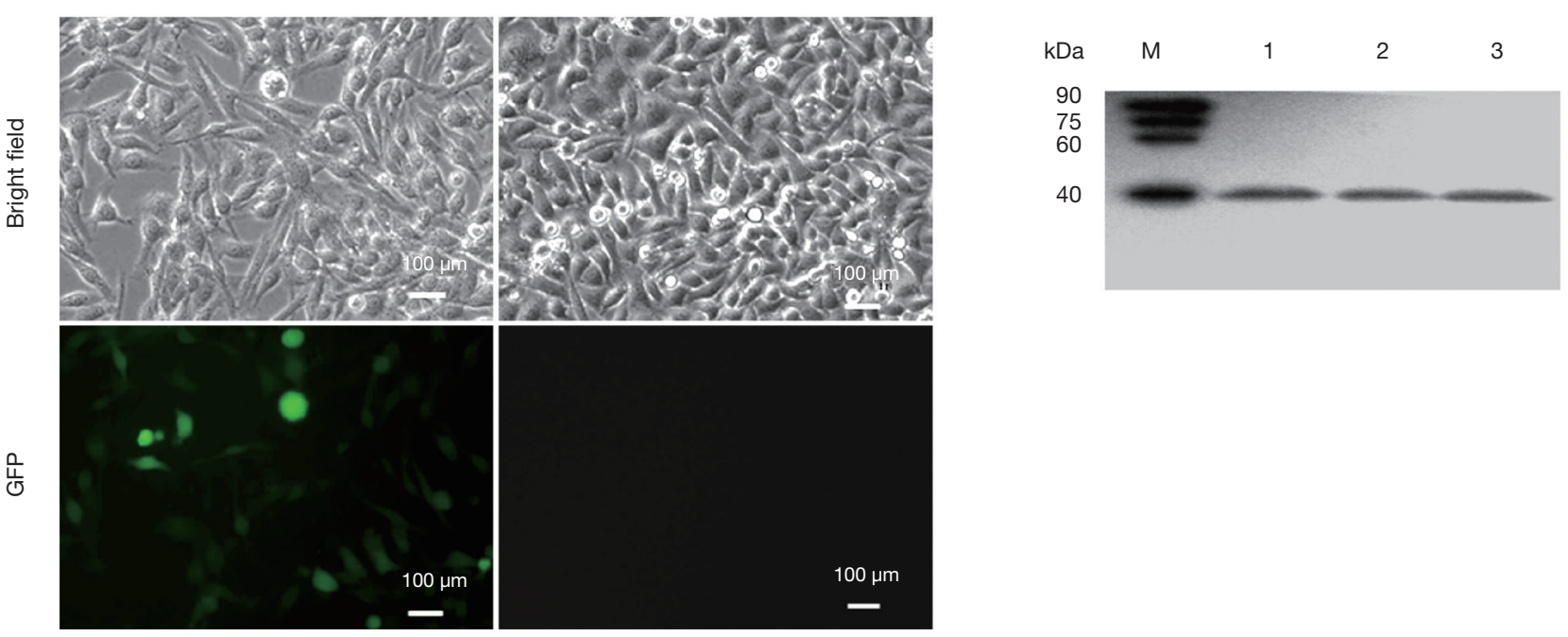

Figure 1 The expression of GFP-pMNSF $\beta$ protein in SUVEC cells was detected. (A) The products of pEGFP-MNSF $\beta$ was digested by ApaIand BglII. (B) GFP-pMNSF $\beta$ protein expression in clonal cell line under G418 selection after 2, 4, and 10 days of incubation observed by inverted fluorescence microscopy. (C) Expression of GFP-pMNSF $\beta$ in clonal cells at passage 40. (D) Western blot analyses of GFPpMNSF $\beta$ fusion protein expression levels in stably expressing GFP-pMNSF $\beta$ SUVEC lines using anti-GFP tag antibodies. Lanes 1, 2, and 3 represent 5, 10, and 40 passages, respectively. GFP, green fluorescent protein; pMNSF $\beta$, porcine monoclonal non-specific suppressor factor $\beta$; EGFP, enhanced green fluorescent protein.

biological processes (17-19). Furthermore, MNSF $\beta$ was found to bind to distinct lysine residues of specific target proteins, including BCL-G; this binding inhibited activation of ERK proteins, which regulate MAPK pathways $(5,11)$. MNSF $\beta$ has also been shown to conjugate with endophilin
II and to regulate phagocytosis $(1,8)$; it is also known to play a vital role in immune regulation and apoptosis, but until now, studies on MNSF $\beta$ function in porcine models have been scant and unclear. Porcine model systems have close phylogenetic relationships and physiological 
A
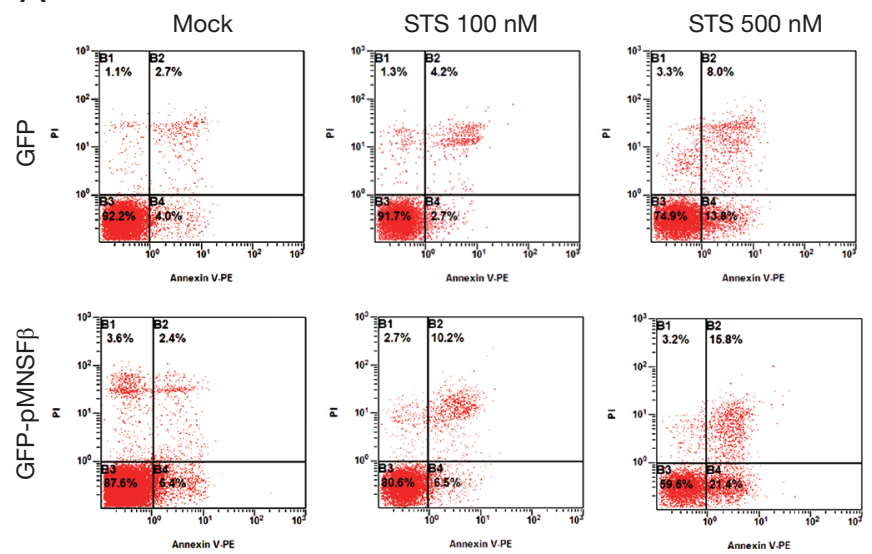

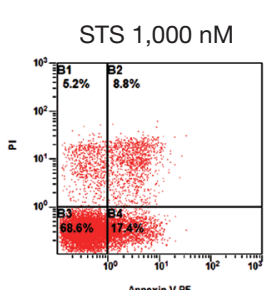

B

Figure 2 Quantification of apoptosis was enhanced by overexpression of GFP-pMNSF $\beta$. (A) Representative FCM diagrams of SUVEC lines stably expressing GFP-vector or GFP-pMNSF $\beta$ were treated for 18 hrs with the STS doses indicated on the $\mathrm{x}$-axis. (B) Annexin V-positive cells were quantified. All experiments were performed in triplicate, and data are expressed as mean \pm SD ( $n=3$ ). Error bars represent standard deviations of replicate data points. ${ }^{* *}, \mathrm{P}<0.01$ and ${ }^{* * *}, \mathrm{P}<0.0001$ versus the control groups (cells stably expressing GFP-vector proteins). FCM, flow cytometry method; SUVEC, swine umbilical vein endothelial cell; STS, staurosporine.
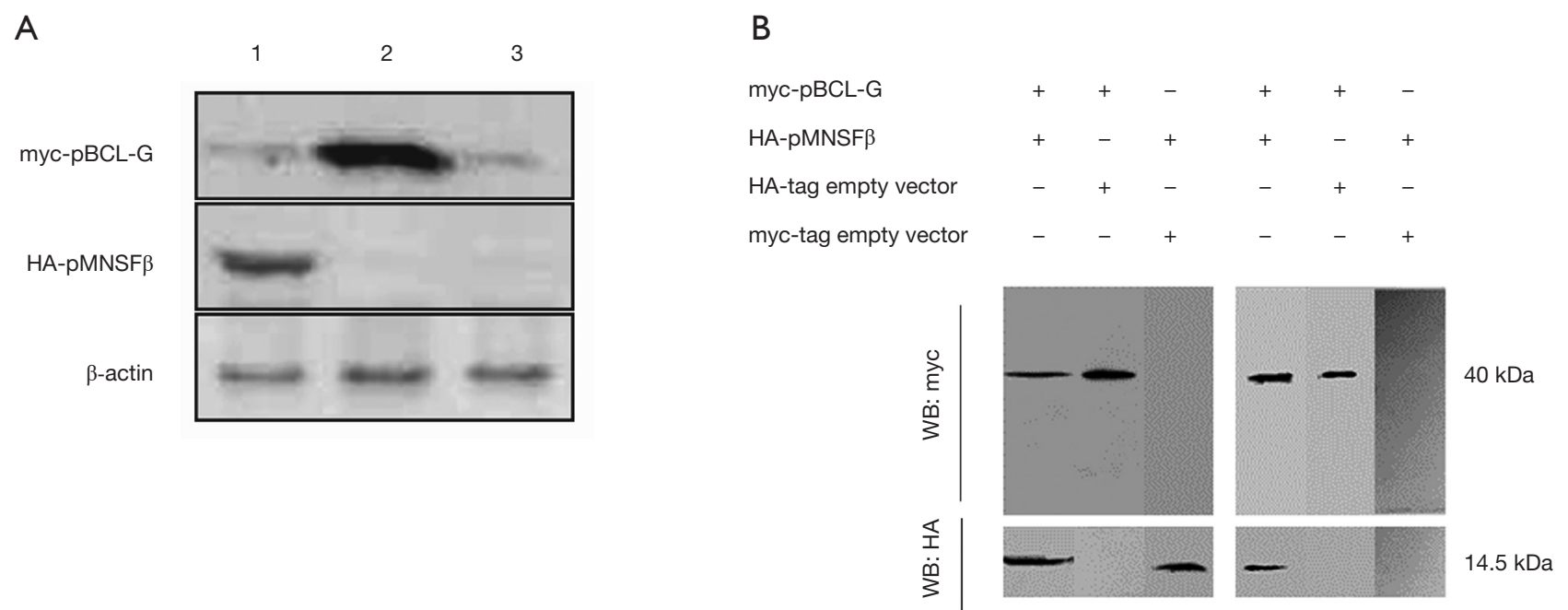

Figure 3 Interaction of HA-pMNSF $\beta$ with myc-pBCL-G was detected by co-immunoprecipitation. (A) Detection of HA-pMNSF $\beta$ and myc-pBCL-G fusion protein expressed in cells by western blot; line 1, 2, and 3 represent SUVEC cells transiently transfected with pCMVHA-pMNSF $\beta$ plasmid, SUVEC cells transiently transfected with pCMV-myc-pBCL-G plasmid, and SUVEC cells, respectively. (B) The individual and co-expressions of HA-pMNSF $\beta$ and myc-pBCL-G proteins in transfected SUVEC cells were examined by western blot analyses. HA, HA-tagged.

similarities with humans (15), so porcine systems have often been considered preferable to mice for modeling human conditions. In this study, we constructed stable SUVEC lines that overexpressed GFP-pMNSF $\beta$ proteins. These lines are now available for use as convenient molecular tools for future experimental studies of $\mathrm{pMNSF} \beta$ functions.

Studies of MNSF $\beta$ in porcine cell lines offer potential molecular models for investigations of human MNSF $\beta$ related diseases. In humans, Watanabe et al. found that MNSF $\beta$ and BCL-G proteins interacted; these binding 
A

GFP-pBCL-G

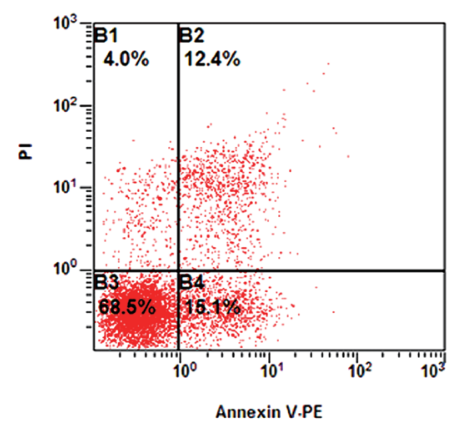

GFP-MNSF $\beta+$ pCMV-myc

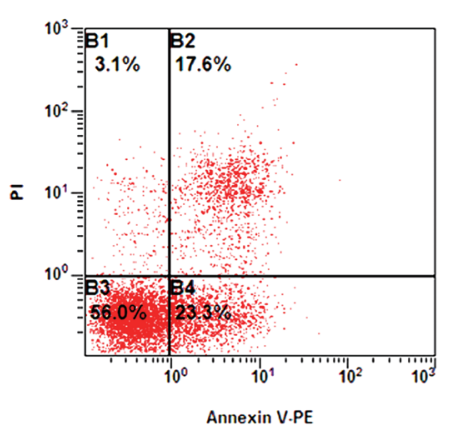

GFP-pMNSF $\beta$

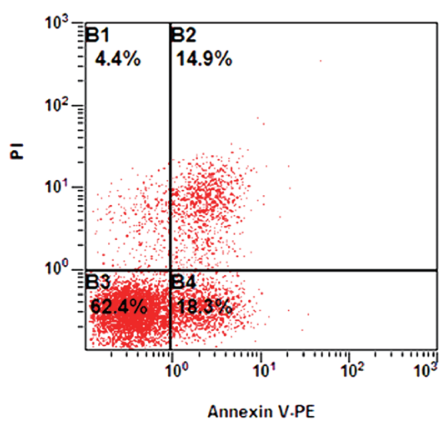

GFP-MNSF $\beta+$ myc-BCL-G

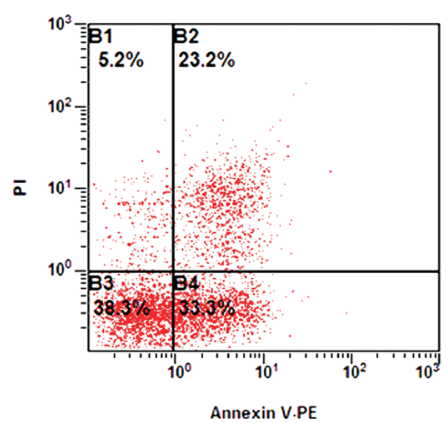

B

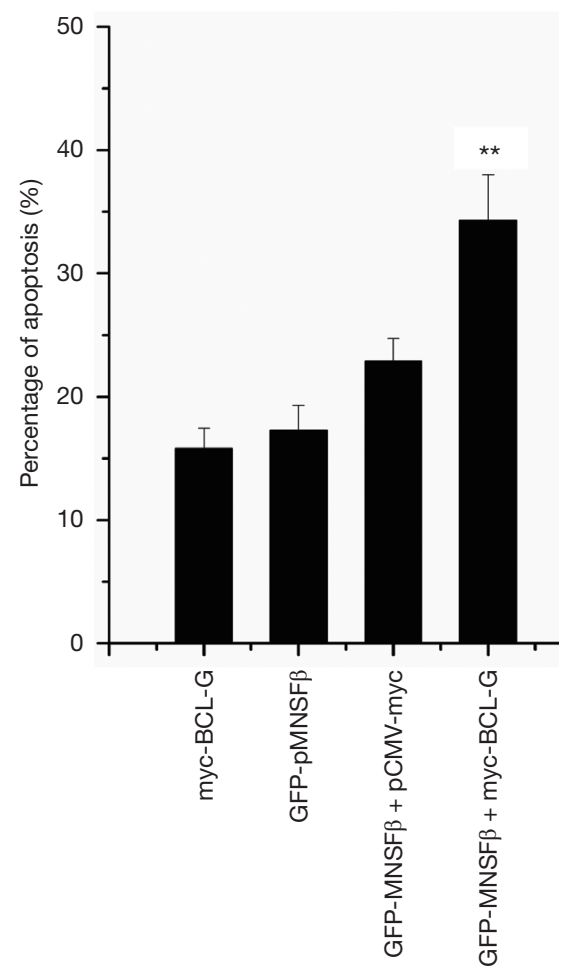

Figure 4 GFP-pMNSF $\beta$ and myc-pBCL-G co-overexpression enhanced STS-induced apoptosis rates. Representative FACS diagrams were given for STS-treated (500 nM) SUVEC lines that stably expressed GFP-pMNSF $\beta$ and were then co-transfected with either myc-pBCL-G or empty vectors. Parent SUVEC lines either transiently expressed myc-BCL-G proteins, or stably expressed GFP-pMNSF $\beta$ and were treated with STS (500 nM) and analyzed as controls. (B) Quantification of Annexin V-positive cells: data, gathered in triplicate, are expressed as the mean \pm SD. Cells co-expressing GFP-pMNSF $\beta$ and myc-BCL-G had significantly more Annexin V-positive events than controls (**, $\mathrm{P}<0.01)$. FACS, fluorescence-activated cell sorting.

events enhanced lipopolysaccharide (LPS)/interferon c-induced apoptosis in macrophages (20). This study showed that overexpression of GFP-pMNSF $\beta$ had proapoptotic effects on SUVEC lines in which apoptosis was induced by varying doses of STS. Together, these previous results and our current study suggest that $\mathrm{pMNSF} \beta$ and human MNSF $\beta$ serve similar functions. Human and porcine MNSF $\beta$ share $93.98 \%$ protein sequence similarities (2). Furthermore, porcine models have been used to study many human conditions including sepsis (21), abdominal aortic aneurysm (22), severe tension pneumothorax (23), and a variety of cancers (24). Thus, porcine models overall, and, specifically, pMNSF $\beta$-expressing SUVEC lines have potential as a tool for characterizing MNSF $\beta$-related diseases and testing drug targets in humans.

The results presented here suggest that $\mathrm{pMNSF} \beta$ is an apoptosis regulatory factor and potential tumor suppressor. Watanabe et al. demonstrated that human MNSF $\beta$ promotes LPS/interferon $\gamma$ (IFN $\gamma$ )-induced apoptosis of Raw264.7 macrophages (20). Statistical analyses of our flow cytometry results demonstrated that overexpression of pMNSF $\beta$ had proapoptotic functions, as it significantly enhanced STSinduced apoptosis in SUVEC lines. This is consistent with previous results. It has been previously reported that overexpression of pBCL-G amplifies STS-induced apoptosis in SUVEC lines (16). Taken together, pMNSF $\beta$ and pBCL-G proteins may similarly function to regulate SUVEC apoptosis. Therefore, $\mathrm{pMNSF} \beta$ gene products may have a new role in the apoptotic regulation and potential tumor suppression of porcine umbilical vein endothelial cells.

Exploration of the specific mechanisms of pMNSF $\beta$ and pBcl-G activities may offer a drastically new direction for the 
treatment of human diseases. BCL-G and MNSF $\beta$ proteins have been demonstrated to interact (20), while BCL-G was shown to regulate apoptosis due to chemotherapy and was found to be misexpressed in systemic lupus erythematosus and congenital heart disease cases (25-28). In this study, we also found that pMNSF $\beta$ interacted with pBCL-G, and co-overexpression synergistically enhanced STS-induced cell apoptosis rates, which was consist with previous studies in humans (20). The present study suggested that $\mathrm{pMNSF} \beta-\mathrm{pBCL}-\mathrm{G}$ complex formation may be important for apoptosis regulation. Future studies exploring specific mechanisms of interaction between MNSF $\beta$ and BCL-G could provide insights and potential treatment options for BCL-G-related human diseases.

In this study, we constructed stably expressing GFP-pMNSF $\beta$ SUVEC lines and stored them in our laboratory. These cell lines were useful and powerful tools for comparative function experiments. We also found that overexpression of $\mathrm{pMNSF} \beta$ proteins significantly enhanced STS-induced apoptosis rates in SUVEC lines, which was consist with previous foundational studies in humans on MNSF $\beta$ function. Additionally, $\mathrm{pMNSF} \beta$ co-immunoprecipitated with pBCL-G, along with overexpression of $\mathrm{pMNSF} \beta$, significantly promoted $\mathrm{pBCL}$ $\mathrm{G}$-induced cell apoptosis. Therefore, pMNSF $\beta$ may be a novel drug target for BCL-G-related human diseases, such as cell apoptosis caused by chemotherapy for cancer, and for systemic lupus erythematosus and congenital heart diseases.

\section{Acknowledgments}

Funding: None.

\section{Footnote}

Reporting Checklist: The authors have completed the MDAR reporting checklist. Available at http://dx.doi.org/10.21037/ atm-20-6348

Data Sharing Statement: Available at http://dx.doi. org/10.21037/atm-20-6348

Conflicts of Interest: All authors have completed the ICMJE uniform disclosure form (available at http://dx.doi. org/10.21037/atm-20-6348). The authors have no conflicts of interest to declare.

Ethical Statement: The authors are accountable for all aspects of the work in ensuring that questions related to the accuracy or integrity of any part of the work are appropriately investigated and resolved. All experiments were conducted in compliance with current Chinese ethical legislation.

Open Access Statement: This is an Open Access article distributed in accordance with the Creative Commons Attribution-NonCommercial-NoDerivs 4.0 International License (CC BY-NC-ND 4.0), which permits the noncommercial replication and distribution of the article with the strict proviso that no changes or edits are made and the original work is properly cited (including links to both the formal publication through the relevant DOI and the license). See: https://creativecommons.org/licenses/by-nc-nd/4.0/.

\section{References}

1. Nakamura M, Shimosaki S. The ubiquitin-like protein monoclonal nonspecific suppressor factor $\beta$ conjugates to endophilin II and regulates phagocytosis. FEBS J 2009;276:6355-63.

2. Wang JN, Jiang P, Kang Z, et al. Cloning, eukaryotic expression and spatial expression patterns of porcine MNSFbeta. Yi Chuan 2013;35:1377-83.

3. Nakamura M, Xavier RM, Tsunematsu T, et al. Molecular cloning and characterization of a cDNA encoding monoclonal nonspecific suppressor factor. Proc Natl Acad Sci U S A 1995;92:3463-7.

4. Kas K, Michiels L, Merregaert J. Genomic structure and expression of the human fau gene: encoding the ribosomal protein S30 fused to a ubiquitin-like protein. Biochem Biophys Res Commun 1992;187:927-33.

5. Nakamura M, Tanigawa Y. Characterization of ubiquitin-like polypeptide acceptor protein, a novel proapoptotic member of the Bcl2 family. Eur J Biochem 2003;270:4052-8.

6. Mahajan R, Delphin C, Guan T, et al. A small ubiquitinrelated polypeptide involved in targeting RanGAP1 to nuclear pore complex protein RanBP2. Cell 1997;88:97-107.

7. Malakhov MP, Kim KI, Malakhova OA, et al. Highthroughput Immunoblotting ubiquitin-like protein isg 15 modifies key regulators of signal transduction. J Biol Chem 2003;278:16608-13.

8. Raasi S, Schmidtke G, Groettrup M. The ubiquitin-like protein FAT10 forms covalent conjugates and induces apoptosis. J Biol Chem 2001;276:35334-43.

9. Hemelaar J, Lelyveld VS, Kessler BM, et al. A single 
protease, Apg4B, is specific for the autophagy-related ubiquitin-like proteins GATE-16, MAP1-LC3, GABARAP, and Apg8L. J Biol Chem 2003;278:51841-50.

10. Marx J. Cell biology. SUMO wrestles its way to prominence in the cell. Science 2005;307:836.

11. Nakamura M, Yamaguchi S. The ubiquitin-like protein MNSF $\beta$ regulates ERK-MAPK cascade. J Biol Chem 2006;281:16861-9.

12. Nakamura M, Watanabe N, Notsu K. Ubiquitinlike protein MNSF $\beta$ covalently binds to cytosolic 10-formyltetrahydrofolate dehydrogenase and regulates thymocyte function. Biochem Biophys Res Commun 2015;464:1096-100.

13. Codas R, Badet L, Eugene M, et al. editors. Evaluation of pulsatile perfusion machine RM3 for kidney preservation in a swine model of renal autotransplantation. Transplant Proc 2009;41:3296-8.

14. Fodor WL, Williams BL, Matis LA, et al. Expression of a functional human complement inhibitor in a transgenic pig as a model for the prevention of xenogeneic hyperacute organ rejection. Proc Natl Acad Sci U S A 1994;91:11153-7.

15. Kozianka J, Kielan W, Waleczek H. Barium peritonitis-a study in pigs. Adv Clin Exp Med 2003;12:569-74.

16. Jiang P, Li D, Bi L, et al. BCL-G as a new candidate gene for immune responses in pigs: Bioinformatic analysis and functional characterization. Vet Immunol Immunopathol 2012;150:112-7.

17. Nakamura M, Tanigawa Y. Ubiquitin-like polypeptide conjugates to acceptor proteins in concanavalin A-and interferon $\gamma$-stimulated T-cells. Biochem J 1998;330:683-8.

18. Haas AL, Ahrens $\mathrm{P}$, Bright $\mathrm{P}$, et al. Interferon induces a 15-kilodalton protein exhibiting marked homology to ubiquitin. J Biol Chem 1987;262:11315-23.

19. Raasi S, Schmidtke G, Do Giuli R, et al. A ubiquitin-like

Cite this article as: Chen $\mathrm{P}$, Wang J, Wang X, Wang Y, Xu C, Ji C. Porcine ubiquitin-like protein MNSF $\beta$ promotes cell apoptosis and covalently binds to BCL-G to enhance staurosporine-induced apoptosis. Ann Transl Med 2020;8(20):1306. doi: 10.21037/atm-20-6348 protein which is synergistically inducible by interferonand tumor necrosis factor. Eur J Immunol 1999;29:4030-6.

20. Watanabe J, Nakagawa $M$, Nakamura $M$, et al. Ubiquitinlike protein MNSF $\beta$ covalently binds to Bcl-G and enhances lipopolysaccharide/interferon $\gamma$-induced apoptosis in macrophages. FEBS J 2013;280:1281-93.

21. Soerensen KE, Olsen HG, Skovgaard K, et al. Disseminated intravascular coagulation in a novel porcine model of severe Staphylococcus aureus sepsis fulfills human clinical criteria. J Comp Pathol 2013;149:463-74.

22. Czerski A, Bujok J, Gnus J, et al. Experimental methods of abdominal aortic aneurysm creation in swine as a large animal model. J Physiol Pharmacol 2013;64:185-92.

23. Nelson D, Porta C, Satterly S, et al. Physiology and cardiovascular effect of severe tension pneumothorax in a porcine model. J Surg Res 2013;184:450-7.

24. Flisikowska T, Kind A, Schnieke A. The new pig on the block: modelling cancer in pigs. Transgenic Res 2013;22:673-80.

25. Benito A, Gutierrez O, Pipaon C, et al. A novel role for proline-and acid-rich basic region leucine zipper (PAR $\mathrm{bZIP}$ ) proteins in the transcriptional regulation of a $\mathrm{BH} 3-$ only proapoptotic gene. J Biol Chem 2006;281:38351-7.

26. O'Connor L, Strasser A, O'Reilly LA, et al. Bim: a novel member of the Bcl-2 family that promotes apoptosis. EMBO J 1998;17:384-95.

27. Lin ML, Park JH, Nishidate T, et al. Involvement of maternal embryonic leucine zipper kinase (MELK) in mammary carcinogenesis through interaction with Bcl-G, a pro-apoptotic member of the Bcl-2 family. Breast Cancer Res 2007;9:R17.

28. Liu X, Pan Z, Zhang L, et al. JAB1 accelerates mitochondrial apoptosis by interaction with proapoptotic BclGs. Cellular Signalling 2008;20:230-40.

(English Language Editor: J. Gray) 